University of Nebraska - Lincoln

DigitalCommons@University of Nebraska - Lincoln

2014

\title{
Cooperative Spectrum Sensing in Cognitive Radio Networks Using Multidimensional Correlations
}

Dongyue Xue

Ohio State University

Eylem Ekici

Ohio State University

Mehmet C. Vuran

University of Nebraska-Lincoln, mcvuran@cse.unl.edu

Follow this and additional works at: https://digitalcommons.unl.edu/csearticles

Xue, Dongyue; Ekici, Eylem; and Vuran, Mehmet C., "Cooperative Spectrum Sensing in Cognitive Radio Networks Using Multidimensional Correlations" (2014). CSE Journal Articles. 138.

https://digitalcommons.unl.edu/csearticles/138

This Article is brought to you for free and open access by the Computer Science and Engineering, Department of at DigitalCommons@University of Nebraska - Lincoln. It has been accepted for inclusion in CSE Journal Articles by an authorized administrator of DigitalCommons@University of Nebraska - Lincoln. 


\title{
Cooperative Spectrum Sensing in Cognitive Radio Networks Using Multidimensional Correlations
}

\author{
Dongyue Xue, Eylem Ekici, and Mehmet C. Vuran
}

\begin{abstract}
In this paper, a multidimensional-correlation-based sensing scheduling algorithm, $(\mathrm{CORN})^{2}$, is developed for cognitive radio networks to minimize energy consumption. A sensing quality metric is defined as a measure of the correctness of spectral availability information based on the fact that spectrum sensing information at a given space and time can represent spectrum information at a different point in space and time. The scheduling algorithm is shown to achieve a cost of sensing (e.g., energy consumption, sensing duration) arbitrarily close to the possible minimum, while meeting the sensing quality requirements. To this end, $(\mathrm{CORN})^{2}$ utilizes a novel sensing deficiency virtual queue concept and exploits the correlation between spectrum measurements of a particular secondary user and its collaborating neighbors. The proposed algorithm is proved to achieve a distributed and arbitrarily close to optimal solution under certain, easily satisfied assumptions. Furthermore, a distributed Selective-(CORN $)^{2}\left(\mathrm{~S}-(\mathrm{CORN})^{2}\right)$ is introduced by extending the distributed algorithm to allow secondary users to select collaboration neighbors in densely populated cognitive radio networks. In addition to the theoretically proved performance guarantees, the algorithms are evaluated through simulations.
\end{abstract}

Index Terms-Cognitive radio networks; cooperative spectrum sensing; correlation; optimal scheduling.

\section{INTRODUCTION}

$\mathbf{T}$ ODAY'S wireless networks are characterized by a fixed spectrum assignment policy. However, a large portion of the assigned spectrum is used sporadically and geographical variations in the utilization of assigned spectrum range from $15 \%$ to $85 \%$ with a high variance in time. The limited available spectrum and the inefficiency in the spectrum usage necessitate a new communication paradigm to exploit the existing wireless spectrum opportunistically. This new networking paradigm is referred to as cognitive radio networks (CRNs) [2]. Based on the ambient spectrum information, cognitive radio users (or secondary users (SUs)) communicate via available channels without disrupting the communication of spectrum owners (or primary user (PUs)).

To assess the spectral availability while maintaining efficient operation of CRNs, effective spectrum sensing solutions are required [2]. Recently, spectrum sensing solutions have been

Manuscript received February 24, 2013; revised June 27 and November 1, 2013; accepted December 31, 2013. The associate editor coordinating the review of this paper and approving it for publication was $\mathrm{M}$. Vu.

D. Xue and E. Ekici are with the Department of Electrical and Computer Engineering, the Ohio State University, Columbus, OH (e-mail: \{xued, ekici\}@ece.osu.edu).

M. C. Vuran is with the Department of Computer Science and Engineering, University of Nebraska-Lincoln, Lincoln, NE (e-mail: mcvuran@cse.unl.edu).

A preliminary version of this paper has appeared in WiOpt 2012 [51]. This work is supported by NSF under grant number CNS-1247941 and CNS1247914.

Digital Object Identifier 10.1109/TWC.2014.022714.130351 developed to provide high detection probability and minimize false alarm rates, where mainly physical layer metrics are considered. In general, spectrum sensing solutions can be classified as cooperative and non-cooperative [2]. More specifically, cooperative solutions rely on multiple SUs to exchange spectrum occupancy information through individual local measurements. This can be achieved through clusterbased architectures [5], wherein the CRN is divided into clusters and each cluster-head makes a decision on the availability of channels. Spectrum availability is assessed by leveraging spectrum utilization information from different cluster heads that receive the local observations of SUs in their clusters. Cooperative sensing schemes are also utilized to estimate the maximum transmit power in cognitive networks so that the interference constraints are satisfied [9].

The existing studies indicate that collaboration among SUs improves the efficiency of spectrum utilization, and allows relaxation of the constraints at individual SUs [12], [25]. However, network-wide effects of spatio-temporal sensing have not been formally analyzed except for heuristics in [25], [26]. While collaboration is shown to improve sensing efficiency at the physical layer, two major tradeoffs exist in terms of network-wide considerations: (1) Cooperative spectrum sensing introduces communication overhead for the dissemination of local observations between SUs. Accordingly, a large number of SUs used for cooperation results in a higher communication cost irrespective of whether a clusterbased or a flat topology is employed. Consequently, energy consumption associated with such communication overhead increases with increasing cooperation. (2) Spectrum utilization observed by closely located SUs is highly correlated due to the inherent spatial correlation in the received PU signals and correlated shadow fading. In addition to the spatial correlation between SUs, the observed information is also correlated in the time domain. More specifically, spectrum information gathered at a particular time represents the spectrum activity at a later time, where the certainty decreases with time difference due to the temporally-correlated nature of the PU activity. If an SU performs local sensing, the additional use of highly correlated spectrum information has minimal effect on improving sensing accuracy [12], [25], [31]. On the other hand, the communication overhead increases regardless. These tradeoffs are generally exploited to limit the number of sensors that collaborate for a spectrum sensing task [30].

The existing work, however, considers local sensing is performed all the time. Instead, we argue that, spectrum sensing information at a given space and time can represent spectrum information at a different point in the space-time 
space. Accordingly, an SU can improve its sensing quality at a particular time by using spectrum information observed by a different $\mathrm{SU}$ at a different time instead of local sensing.

Identifying the main objective of sensing as maintaining a given minimum sensing quality, in this paper, we explore cooperative methods that will minimize the cost of sensing. The cost can flexibly be defined as a means to represent the resources spent (such as energy) or opportunities sacrificed for sensing (such as sensing duration). Since cooperation requires information exchange, these costs will also explicitly incorporate communication activities. Accordingly, we develop a provably arbitrarily close to optimal correlation-based cooperative spectrum sensing algorithm, named (CORN $)^{2}$, through a novel sensing deficiency virtual queue concept and exploit the correlation between SUs. The algorithm further leads to a distributed solution when correlation weights are upper-bounded by some constants (referred in the paper as the bounded contribution assumption), which holds especially in low Signal-to-Noise Ratio (SNR) environments with a high level of temporal correlation of spectrum sensing information. In densely populated CRNs, the communication overhead may reap the benefits of cooperation. To address this issue, the distributed $(\mathrm{CORN})^{2}$ is further extended to a Selective$(\mathrm{CORN})^{2}\left(\mathrm{~S}-(\mathrm{CORN})^{2}\right)$ in densely populated CRNs where $\mathrm{SU}$ nodes are selective in using their neighbor reports on sensing information.

The rest of the paper is organized as follows: In Section II, related work in spectrum sensing correlation and utilityoptimal scheduling in wireless networks is discussed. The problem description and the models used to represent spectrum sensing are introduced in Section III. We introduce $(\mathrm{CORN})^{2}$, an arbitrarily close to optimal correlation-based cooperative spectrum sensing scheduling algorithm, in Section IV and analyze its theoretical performance in Section V. $(\mathrm{CORN})^{2}$ is further extended for densely populated CRNs in Section VI. A numerical evaluation of $(\mathrm{CORN})^{2}$ is presented in Section VII. The paper is concluded in Section VIII.

\section{RELATED WORK}

Spectrum management in CRNs has recently been investigated in the literature [3]. Most notably, optimal spectrum sensing schemes have been developed for non-cooperative and cooperative spectrum sensing solutions [6], [7], [10], [11], [13]. To this end, recently, spectral correlation is exploited to minimize the spectrum sensing cost without hampering the spectrum sensing accuracy [4], [8]. However, these solutions focus only on the spectral content of the signal received by a single user and do not address spatial correlation between SUs. In addition, spatial correlation between signals received at closely located SUs have been investigated [12], [25], [31]. Furthermore, due to primary signal characteristics, the spectrum activity is also temporally correlated. Correlationbased sensing scheduling algorithms have been proposed in [10], [43], [44], [45], [6], [46], [47], [48], [49], [50]. Algorithms in [10], [43] aim to minimize the network-wide energy consumption employing spatial correlations only. However, the sensing quality constraint (in terms of probability of false alarm and misdetection) is a global one for the CR base station, which requires centralized control. On the other hand, the sensing quality constraint is imposed on local SU nodes in our work. In [44], [45], consensus-based sensing scheduling algorithms are proposed for a cognitive radio mobile ad hoc network, but local sensing is performed all the time. On the other hand, in our work, SUs can choose to improve their sensing quality at a particular time by using spectrum information observed by a different SU instead of local sensing. Spatial correlation is considered in the optimal spectrum sensing algorithm in [6]. However, the correlation is assumed to be 1 , which does not apply to the setting where spatial correlation diminishes over the distance between SUs. In [46], while temporal correlation on PU activity is considered, the spectrum access algorithm is proposed for a single-SU singlePU scenario. The algorithm in [46] is not readily extendable to a CRN composed of multiple cooperative SUs. While spatial correlations are considered in [47], local sensing is performed all the time. A sensing scheduling algorithm is proposed in [48] considering temporal and spatial correlations via a game theory approach, but the objective is very different from that of our work. Specifically, a network-wide utility is maximized given energy consumption constraint in [48]. In contrast, we minimize energy expenditure given local sensing and sensing quality constraint. In [49], spatial-temporal correlations are employed in the optimal opportunistic spectrum access for a mobile cognitive radio network. However, the objective is to maximize SU throughput, which is different from our objective of minimizing energy consumption. In [50], temporal correlations are exploited to optimally detect (in terms of probability of misdetection and probability of false alarm) the existence of PU. However, local sensing is required for all the secondary receivers in the CRN.

The sensing problem investigated in this paper is a utility optimal scheduling problem with energy minimization. Throughput/utility-optimal scheduling for wireless communication networks has been studied in the past in detail. The seminal work on back-pressure-based scheduling [14] and its extensions have been widely employed in developing optimal scheduling in wireless networks. Throughput/utility-optimal routing and scheduling algorithms have been developed in [16], [17], [18]. These optimal scheduling algorithms are generally computationally prohibitive and impractical for distributed implementations. Distributed algorithms are proposed in [19], [20], [21], [22] at the sacrifice of throughput/utility optimality. In this work, we show that when the bounded contribution assumption holds (i.e., correlation weights are appropriately bounded above), the proposed algorithm can achieve both distributed implementation and optimality of cost of sensing.

\section{PRELIMINARIES}

\section{A. Objective and Motivation}

Information about spectral availability is the key for a healthy CRN. We define the Sensing Quality as a measure of the correctness of spectral availability information. An instance of sensing quality can be formulated as a combination of false alarm and mis-detection probabilities of a particular sensing algorithm. Under our framework, the sensing quality is a dynamic measure of spectral availability information. It 
varies not only based on the inherent limitations of individual sensors (e.g., ability to distinguish PU channel usage from noise), but also as a function of time and the origin of the information. For the sake of simplicity, consider a perfectly accurate spectral sensing result generated by a given node. Traditionally, a rigid period of validity is assumed for such information. In fact, the validity of information can be represented as a non-increasing function of time unless it is augmented with new measurements. Moreover, if the sensing information is shared in a neighborhood, its ability to represent the correct channel availability information generally diminishes with distance as well as time. As such, we envision that every cognitive node will strive to maintain a minimum sensing quality level to ensure proper operation. In the following, we introduce the models to represent spectrum sensing.

\section{B. Spectrum Sensing Quality}

Consider a network with a PU and a set of SUs $\mathcal{N}$, where $N_{s}=|\mathcal{N}|$, and the wireless spectrum $\mathcal{S}$ is divided into spectrum bands (channels) $c$, where $c \in \mathcal{S}$ and $N_{c}$ is the number of channels. Any one of these channels can be occupied by a PU at any time. Accordingly, the activity of a PU in a channel $c$, at location $\mathbf{s}_{p}$ and time $t$ is denoted as a binary variable $A_{p}\left(c, \mathbf{s}_{p}, t\right)$, which is equal to 1 if the $\mathrm{PU}$ is transmitting.

We consider a spectrum sensing mechanism, where each SU $j$ samples the energy of a set of channels $\mathcal{C}_{j} \subseteq \mathcal{S}$ using energy detection. Accordingly, the received signal sampled by an $\mathrm{SU} j$ at location $\mathbf{s}_{j}$ is denoted by $Y_{j}\left[c, n_{j}\right]=y\left(c, \mathbf{s}_{j}, t_{n_{j}}\right)$ for a channel $c \in \mathcal{C}_{j}$ at discrete time $t_{n_{j}}=n_{j} T_{u}$, where $T_{u}$ is unit time. Assuming all powers are normalized according to the transmit power of the primary transmitter and the PU resides at the center of the coordinate system, the received energy sampled by the SU $j$ can be modeled as follows:

$$
\begin{aligned}
y\left(c, \mathbf{s}_{j}, t_{n_{j}}\right) & =A_{s}\left(c, \mathbf{s}_{j}, t_{n_{j}}\right)+W, \\
A_{s}\left(c, \mathbf{s}_{j}, t_{n_{j}}\right) & =\alpha\left(c, \mathbf{s}_{j}, t_{n_{j}}\right) A_{p}\left(c, \mathbf{0}, t_{n_{j}}\right)
\end{aligned}
$$

where $A_{s}\left(c, \mathbf{s}_{j}, t_{n_{j}}\right)$ is the attenuated version of the PU signal, $A_{p}\left(c, \mathbf{0}, t_{n_{j}}\right)$, at the location of the $\mathrm{SU} j, \mathbf{s}_{j}$, at time $t_{n_{j}}$; $\alpha\left(c, \mathbf{s}_{j}, t_{n_{j}}\right)$ models the attenuation and fading in the channel; $\mathbf{0}=\{0,0\}$ is the location of the PU, and $W \sim \mathcal{N}\left(0, \sigma_{N}\right)$ is the detection noise at the SU. Note that in the model (1), we assume that the propagation delay is negligible.

Now consider another SU $i$ at location $\mathbf{s}_{i}$ that performs spectrum sensing for the same channel $c$ at time $t_{n_{i}}\left(t_{n_{i}}>\right.$ $\left.t_{n_{j}}\right)$. Through collaboration, the SU $i$ can use the sensing information of SU $j$ at a different time and location for spectrum sensing. Due to the correlation in spatial and temporal dimensions, the sensed information at SUs $i$ and $j$ are correlated. More specifically, the spatio-temporal correlation function $\rho(\cdot)$ for two channel samples taken in channel $c$; at locations $\mathbf{s}_{i}$ and $\mathbf{s}_{j}$; and at times $t_{n_{i}}$ and $t_{n_{j}}$ is given by

$$
\rho\left(\Delta_{i, j}^{s}, \Delta_{i, j}^{t}\right) \triangleq \frac{E\left[Y_{i}\left[c, n_{i}\right] Y_{j}\left[c, n_{j}\right]\right]}{\sigma_{Y}^{2}}=\rho_{s}\left(\Delta_{i, j}^{s}\right) \cdot \rho_{t}\left(\Delta_{i, j}^{t}\right)
$$

where $\sigma_{Y}^{2}$ is the variance of the signal, $\Delta_{i, j}^{s}=\left\|\mathbf{s}_{i}, \mathbf{s}_{j}\right\|$, and $\Delta_{i, j}^{t}=\left|t_{n_{i}}-t_{n_{j}}\right|$ are the differences in spatial and temporal dimensions. Without loss of generality, we model the correlation function with spatial $\left(\rho_{s}(\cdot)\right)$ and temporal $\left(\rho_{t}(\cdot)\right)$ components. In the following, we explain their compositions.

- Spatial Correlation: In RF signal measurements, the spatial correlation between observed samples occurs because of the correlated nature of shadow fading [32]. Theoretical and experimental studies show that there is an exponential relationship between spatial correlation and the distance between sample points [26], [27], [28], [33].

- Temporal Correlation: Temporal correlation between spectrum samples can be modeled as small-scale and large-scale temporal correlation. Small-scale temporal correlation exists due to the primary user signal properties. For a high enough sampling rate, observed samples are correlated since the primary user signal is correlated. On the other hand, large-scale temporal correlation exists due to the movement of the secondary user [32]. As the secondary user moves within the spatially correlated signal field, the spectrum samples become correlated. This correlation is directly proportional to the sampling rate but inversely proportional to the speed of the SU [34].

Recent long-term and large-scale spectrum sensing experiments also confirm the existence of correlation in space and time [35]. Small-scale temporal correlation is generally exploited within spectrum sensing algorithms in terms of sample averaging or cyclostationary feature analysis as a means to improve sensing accuracy. This correlation is negligible when the time difference between different spectrum sensing attempts are considered. Consequently, we model the spatial and temporal correlation in spectrum sensing in (2) as follows [33]:

$$
\rho_{s}\left(\Delta_{i, j}^{s}\right)=e^{-\frac{\Delta_{i, j}^{s}}{D_{\text {corr }}}} ; \quad \rho_{t}\left(\Delta_{i, j}^{t}\right)=e^{-\frac{\Delta_{i, j}^{t} v_{i}}{D_{\text {corr }}}}
$$

where $D_{c o r r}$ is the decorrelation distance and $v_{i}$ is the speed of the SU. Note that since the correlation of the underlying stochastic process is still due to location, the correlation distances are the same for both spatial and temporal correlations. Hence, they follow the same spatial correlation parameter, i.e., $D_{\text {corr }}$.

Now, consider a multi-user spectrum sensing setting, where a node $i$ performs spectrum sensing according to the following observation definition:

$$
T_{i}\left(c, n_{i}\right)=\sum_{j \in \mathcal{I}_{i}} w_{j, i}^{c}\left(n_{i}\right) Y_{j}\left[c, n_{j}\right],
$$

where $\mathcal{I}_{i}$ is the set of neighbors of $i$, including the node $i$, within which cooperative sensing can be performed, $w_{j, i}^{c}\left(n_{i}\right)$ is the weight factor, and $Y_{j}\left[c, n_{j}\right]$ is the most recent observation of one of the neighbors $j$ at discrete time $n_{j}\left(n_{j} \leq n_{i}\right)$. If non-cooperative sensing is employed, $w_{j, i}^{c}\left(n_{i}\right)=0, \forall j \neq i$ and $w_{i, i}^{c}\left(n_{i}\right)=1$. For cooperative sensing, the weight factor, $w_{j, i}^{c}\left(n_{i}\right)$, can be selected according to the correlation between spectrum samples as modeled in (2) and (3). A more detailed model of $w_{j, i}^{c}\left(n_{i}\right)$ can be found in [51].

It is clear that it takes a relatively long time to sense the whole spectrum in a wide band cognitive radio network. Instead, it might be more efficient to share channels to be 
sensed among neighbors, which have correlated observations. Intuitively, if the local observation of node $i$ on a particular channel is highly correlated with one of its neighbors, the node may use the neighbor's information only without any local sensing on that channel. Such cooperation decreases the cost for spectrum sensing. On the other hand, if node $i$ performs local sensing on a channel, any information from its closest neighbors would be highly correlated and will not improve sensing accuracy [28]. Existing work so far has assumed all the information is available at an SU or a fusion center and the correlation aspects are considered accordingly. In that case, communication overhead increases to exchange local observations. With the notion of spectrum representativeness, this overhead can be mitigated. Next, we propose a novel model to capture this tradeoff in correlated sensing performance.

\section{Correlation-based CoOperative Spectrum SEnsing SCHEduling Algorithm ((CORN $\left.)^{2}\right)$}

In this section, we model the CRN from the perspective of individual SUs and their requirements of sensing quality. Accordingly, we develop a provably arbitrarily close to optimal sensing scheduling algorithm through a novel sensing deficiency virtual queue concept and introduce its distributed implementation.

\section{A. Problem Definition}

Let an SU $i$ have access to a set of communication channels $\mathcal{C}_{i} \subseteq \mathcal{S}$. For sensing accuracy, we associate each pair of SU $i$ and channel $c, c \in \mathcal{C}_{i}$, with a minimum rate of sensing quality $R_{D}$ that needs to be maintained at all times. This minimum level can be achieved by sensing the channel locally and/or by exchanging spectrum sensing reports between other SUs in the vicinity. The sensing quality is assumed to decay in time at a constant rate, and needs to be supplemented with additional sensing data. The cooperative nature of this framework stems from sharing the sensing information within $\mathcal{I}_{i}$ neighborhoods. In addition to cooperation, each SU $i$ must also sense a channel $c$ locally at a minimum rate of $R_{S}{ }^{1}$ This requirement forces each SU $i$ to participate in sensing above a minimum rate and not rely solely on other nodes' observations. For analytical simplicity, we assume $R_{D}$ and $R_{S}$ are constants. The analysis can be easily extended to the case where $R_{D}$ and $R_{S}$ change over SUs and channels (i.e., $R_{D}$ and $R_{S}$ can be replaced with $R_{D}(i, c)$ and $\left.R_{S}(i, c), i \in \mathcal{N}, c \in \mathcal{C}_{i}\right)$.

We define $\mu_{i, c}(t) \in\{0,1\}$ as an indicator of the sensing event of SU $i$ over channel $c$ at time $t . \mu_{i, c}(t)$ can also be considered as an integer value in $\{0,1\}$ corresponding to the normalized quality of sensing. That is, when an SU $i$ senses a channel $c$ at a discrete time $t$, this event contributes to its sensing quality by $\mu_{i, c}(t)$. On the other hand, when the sensing information of another $\mathrm{SU} j$ is used, its contribution is scaled by a factor of $w_{j, i}^{c}(t)$, the correlation weight, which captures

\footnotetext{
${ }^{1}$ We assume that all the SUs remain silent during the sensing interval Compared to conventional sensing solutions, however, during the same sensing interval, SUs can observe different channels. Consequently, an SU can utilize the spectrum sensing results of a particular channel at a different time.
}

the representativeness of $j$ 's sensing data about channel $c$ at $i$. It can be computed using methods outlined in Section III-B, according to the spatial temporal correlations.

Here, it is important to identify the differences between $\mu_{j, c}(t)$ and $w_{j, i}^{c}(t)$. When a node $j$ decides to sense channel $c$ at time $t$, the sensing process contributes to its sensing quality by $\mu_{j, c}(t)$, which is related to the accuracy of the local sensing algorithm. Following sensing, this information is broadcasted. If a neighbor $i$ uses node $j$ 's sensing information, the contribution of node $j$ 's sensing information to node $i$ 's sensing is further scaled by $w_{j, i}^{c}(t)$, which is related to the spatial and temporal correlation between node $i$ and $j$ 's observations.

At any given discrete point in time $t$, a node $i$ improves its sensing quality about a channel $c$ by

$$
M_{i, c}(t) \triangleq \min \left\{\sum_{j \in \mathcal{I}_{i}} \mu_{j, c}(t) w_{j, i}^{c}(t), M_{i, c}^{\max }\right\},
$$

where $M_{i, c}^{\max }>1$ represents the normalized maximum level of information that $i$ can obtain about $c$ 's state, and $w_{i, i}^{c}(t) \triangleq 1$. The linear combination approximation of sensing quality holds especially in low SNR environments, where the PU signal is potentially sensed from sources located far from SUs, such as TV transmitters, and potential PU receivers are nearby, such as TV receivers. We also associate each local sensing event with a fixed cost $P_{S}$. While, in general, a node is not required to broadcast its sensing data, in a cooperative setting, we assume that an SU always broadcasts the result to its neighboring nodes at the cost of $P_{T x}$. An SU receives this information at the cost of $P_{R x}$. The parameters, $P_{S}, P_{T x}$, and $P_{R x}$, can readily be associated with energy consumption for the respective activities. In every discrete time instant, the cost $G_{i}(t)$ is computed as

$$
G_{i}(t)=\sum_{c \in \mathcal{C}_{i}}\left[\left(P_{S}+P_{T x}\right) \mu_{i, c}(t)+\sum_{j \in \mathcal{I}_{i} \backslash\{i\}} P_{R x} \mu_{j, c}(t)\right] .
$$

We would like to find the best scheduling policy $\Omega^{*}=$ $\left\{\mu_{i, c}(t)\right\}_{i, c, t}$ to minimize the cost while satisfying the sensing quality requirements.

\section{Problem Formulation:}

Consider a CRN with SUs $i \in \mathcal{N}$. Find an optimal sensing scheduling policy $\Omega^{*}$ such that the network-wide cost is minimized while all individual sensing quality requirements are satisfied:

$$
\operatorname{minimize} \lim _{T \rightarrow \infty} \frac{1}{T} \sum_{t=0}^{T-1} \sum_{i \in \mathcal{N}} G_{i}(t)
$$

$$
\begin{aligned}
\text { subject to } \lim _{T \rightarrow \infty} \frac{1}{T} \sum_{t=0}^{T-1} \mu_{i, c}(t) \geq R_{S} \\
\text { and } \lim _{T \rightarrow \infty} \frac{1}{T} \sum_{t=0}^{T-1} M_{i, c}(t) \geq R_{D}, \forall i \in \mathcal{N}, c \in \mathcal{C}_{i},
\end{aligned}
$$

where $\sum_{c \in \mathcal{C}_{i}} \mu_{i, c}(t) \leq K$ for a given $i$ and $t$, i.e., an SU can sense up to $K$ channels at any point in time (Correspondingly, the maximum time during which the SUs should be silent is 
upper-bounded by $K$ times the sensing interval for a single channel).

Note that the above problem formulation aims to achieve minimum sensing quality rates asymptotically. While the first constraint in (6) forces each node to perform local sensing, the second constraint in (6) encourages collaboration. The problem can further be refined by including the decision to share or withhold sensing information, as well. For the sake of tractability, first, we limit the discussion to always sharing cases. Then, in Section VI, we relax this assumption such that SUs can decide whether to receive sensing information of a neighbor. Similarly, we assume that the broadcasting of information can be performed in a lossless manner with the help of the scheduling algorithm.

For technical simplicity, we introduce the following assumptions that will assist us in developing the sensing scheduling algorithm:

Assumption 1: We assume that $w_{i, j}^{c}(t)$ is fixed over any time slot duration and i.i.d. over time slots, with values taken from a finite set.

Assumption 2: We assume that the cooperation between any two nodes is fair, i.e., for any channel $c, \forall i, j \in \mathcal{N}$, we have: $c \in \mathcal{C}_{i}$ and $i \in \mathcal{I}_{j}$ if and only if $c \in \mathcal{C}_{j}$ and $j \in \mathcal{I}_{i}$.

Assumption 3: We assume that the sensing quality improved over any time slot is upper-bounded by some constant $M_{\max }$ : $\sum_{j \in \mathcal{I}_{i}} \mu_{j, c}(t) w_{j, i}^{c}(t) \leq M_{\max }, \forall i \in \mathcal{N}, \forall c \in \mathcal{C}_{i}$. Note that this assumption is valid, since we can letx $M_{\max } \triangleq \max _{i, c} M_{i, c}^{\max }$.

\section{B. Optimal Correlation-Based Cooperative Spectrum Sensing Scheduling Algorithm $\left((\mathrm{CORN})^{2}\right)$}

To solve the minimum-cost sensing scheduling problem, we present a novel virtual deficiency queue concept, where the sensing dynamics are represented with virtual queuing structures, operating in discrete time domain. We define two types of per-node, per-channel virtual queues that track the dynamics of sensing quality as shown in Fig. 1. The first virtual local sensing queue $Q_{i, c}^{S}(t)$ (Fig. 1(a)) represents the local sensing events for channel $c$ in SU $i$ with a periodic arrival of "packets" at rate $R_{S}$ and an instantaneous service rate of $\mu_{i, c}(t)$. The evolution of the local sensing queue follows, $\forall i \in \mathcal{N}, c \in \mathcal{C}_{i}$,

$$
Q_{i, c}^{S}(t+1)=\left[Q_{i, c}^{S}(t)+R_{S}-\mu_{i, c}(t)\right]^{+},
$$

where $[a]^{+} \triangleq \max \{a, 0\}$. A "packet" arrival to the local sensing queue represents an increase in need for local sensing, which is satisfied when the packet is "served" and departs from the queue, i.e., node performs local sensing.

The second virtual queue is the total sensing deficiency queue $Q_{i, c}^{D}(t)$ (Fig. 1(a)) for channel $c$ at SU $i$ with a periodic arrival of "deficiency packets" at rate $R_{D}$ and instantaneous service rate of $M_{i, c}(t)$. Each deficiency packet arrival to $Q_{i, c}^{D}(t)$ represents a decay in sensing quality. The decay in sensing quality is countered by the departure of "deficiency packets", which corresponds to the improvement of the sensing quality. In general, a large value of $Q_{i, c}^{D}(t)$ corresponds to a large sensing deficiency, i.e., a low sensing quality. With local sensing events and sensing reports gathered from neighbors, deficiency packets are "served", which causes the $Q_{i, c}^{D}$ to shrink. This, in turn, corresponds to an improved sensing quality for the given SU-channel pair. The evolution of the total sensing deficiency queue follows, $\forall i \in \mathcal{N}, c \in \mathcal{C}_{i}$,

$$
Q_{i, c}^{D}(t+1)=\left[Q_{i, c}^{D}(t)+R_{D}-M_{i, c}(t)\right]^{+} .
$$

When a sensing scheduling algorithm stabilizes the virtual queues $Q_{i, c}^{S}(t)$ and $Q_{i, c}^{D}(t)$, then this means that their respective arrival rates are smaller than or equal to their average service rates, i.e., the two conditions of the general problem formulation in (6) are satisfied. Therefore, when a feasible algorithm stabilizes the system, the constraints of the optimal sensing scheduling problem are automatically satisfied. Consequently, the system parameters $R_{S}$ and $R_{D}$ appear in the system model as arrival rates to our virtual queue structures. Without loss of generality, we assume that arrivals to the virtual queues occur periodically with rates $R_{S}$ and $R_{D}$, respectively.

At this point, we note that the sensing scheduling algorithm also bears significant differences from earlier works on power allocation: In single-channel power-optimal scheduling problem [15], [23], the channel state is known a priori to a node in its interference range, whereas in the power optimal sensing scheduling algorithm, multiple neighboring nodes are required to sense channels and share the information among themselves. Moreover, while a packet transmission has a direct effect on the reduction of queues (namely, on the queues of the transmitting node), a sensing event reduces the deficiency queues of multiple nodes in the neighborhood. Since a service decision for a node affects more than one other neighbor, the solution structure is completely different than traditional scheduling solutions.

The arbitrarily close to optimal cooperative sensing scheduling algorithm that solves the target problem is given below. Its optimality is proven in Section V.

\section{Correlation-Based Cooperative Spectrum Sensing Scheduling Algorithm $\left((\mathrm{CORN})^{2}\right)$ :}

Consider a CRN with SUs $i \in \mathcal{N}$. For each time slot $t$,

$$
\begin{gathered}
\max _{\left(\mu_{i, c}(t)\right)} \sum_{i \in \mathcal{N}} \sum_{c \in \mathcal{C}_{i}}\left[\mu_{i, c}(t) \times\right. \\
\left(Q_{i, c}^{S}(t)-V\left(P_{S}+P_{T x}+P_{R x}\left(I_{i}^{c}-1\right)\right)\right) \\
\left.\quad+\left(\min \left\{\sum_{j \in \mathcal{I}_{i}} \mu_{j, c}(t) w_{j, i}^{c}(t), M_{i, c}^{\max }\right\}\right) Q_{i, c}^{D}(t)\right], \\
\text { s.t. } \sum_{c \in \mathcal{C}_{i}} \mu_{i, c}(t) \leq K, \forall i \in \mathcal{N},
\end{gathered}
$$

where $V$ is a control parameter which trades off between the optimality and the virtual queue backlogs (to be discussed in detail in Remark 2 in Section V-A). In (9), $I_{i}^{c} \triangleq \sum_{j \in \mathcal{I}_{i}} \mathbf{1}_{c \in \mathcal{C}_{j}}$, i.e., the number of neighboring SUs that can contribute to SU $i$ 's sensing quality of channel $c$, including the node $i$. We note that $\mu_{i, c}(t)$ and $M_{i, c}(t)$ are the service rates of queue local sensing queue $Q_{i, c}^{S}(t)$ and the total sensing deficiency queue $Q_{i, c}^{D}(t)$, respectively from (7)(8). With $Q_{i, c}^{S}(t)$ weighted by $\mu_{i, c}(t)$ and $Q_{i, c}^{D}(t)$ weighted by $M_{i, c}(t)$ in (9), the intuition behind the $(\mathrm{CORN})^{2}$ algorithm is: when the virtual queue is congested, the corresponding service rate is more likely to be larger. We will show in Section $\mathrm{V}$ that $(\mathrm{CORN})^{2}$ achieves an 


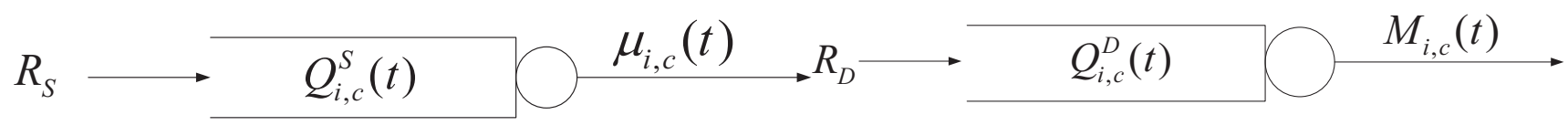

(a) Local Sensing Queue

(b) Total Sensing Deficiency Queue

Fig. 1. Virtual queue structures for sensing quality control.

overall sensing cost arbitrarily close to the minimum, while stabilizing $\left(Q_{i, c}^{S}(t)\right)$ and $\left(Q_{i, c}^{D}(t)\right)$, i.e., constraints (6) are satisfied.

This algorithm requires network-wide information to compute the sensing schedules for each SU. It can be executed centrally by a cluster head if the SUs are organized into (multi-hop or single-hop) clusters. However, it is possible for individual SUs to compute their own sensing schedules using information only from their cooperation neighborhood $\mathcal{I}_{i}$ under certain conditions as will be discussed next.

\section{Distributed Solution under Bounded Contribution Assump- tion}

The general algorithm of (9) uses a cap on the maximum amount of information that can be obtained in a sensing cycle, which is represented as $M_{i, c}^{\max }$. While this quantity has practical significance by not allowing arbitrary levels of accuracy to "accumulate" within a sensing cycle, it also complicates the algorithm execution. This parameter can be removed from consideration when correlation weights are appropriately upper-bounded such that the bounded contribution assumption holds:

$$
\sum_{j \in \mathcal{I}_{i}} \mu_{j, c}(t) w_{j, i}^{c}(t) \leq M_{i, c}^{\max }, \forall i \in \mathcal{N}, \forall c \in \mathcal{C}_{i}, \forall t
$$

Since $\left(\mu_{j, c}(t)\right)$ and $\left(w_{j, i}^{c}(t)\right)$ are upper-bounded, this assumption can be easily satisfied if $M_{i, c}^{\max }$ is sufficiently large. This assumption especially holds in low SNR environments and when the temporal correlation levels of spectrum sensing information are high (i.e., $M_{i, c}^{\max }$ is sufficiently large). Under this assumption, the optimization (9) can be simplified as follows:

$$
\begin{gathered}
\max _{\left(\mu_{i, c}(t)\right)} \sum_{i \in \mathcal{N}} \sum_{c \in \mathcal{C}_{i}} \mu_{i, c}(t)\left[Q_{i, c}^{S}(t)+\sum_{j \in \mathcal{I}_{i}} w_{i, j}^{c}(t) Q_{j, c}^{D}(t)\right. \\
\left.-V\left(P_{S}+P_{T x}+P_{R x}\left(I_{i}^{c}-1\right)\right)\right],
\end{gathered}
$$

subject to the channel sensing constraints (10). (12) is a typical maximal weight matching problem on the bipartite graph formed by the node set $\mathcal{N}$ and the channel set $\mathcal{S}$. Since the matching weight $\left[Q_{i, c}^{S}(t)+\sum_{j \in \mathcal{I}_{i}} w_{i, j}^{c}(t) Q_{j, c}^{D}(t)-V\left(P_{S}+\right.\right.$ $\left.\left.P_{T x}+P_{R x}\left(I_{i}^{c}-1\right)\right)\right]$ in (12) can be obtained locally for each $\mathrm{SU} i$ and channel $c$, the optimization problem can be optimally solved in a greedy and distributed manner. Specifically, each SU $i \in \mathcal{N}$ chooses the first $\min \left\{\left|\mathcal{C}_{i}\right|, K\right\}$ channels with largest positive matching weights over which SU $i$ performs sensing, with a time complexity of $O\left(N_{c}+\min \left\{\left|\mathcal{C}_{i}\right|, K\right\} \log N_{c}\right)$.

Remark 1: In each time slot, the optimization problem (12) can be solved locally and optimally by each SU using the sensing deficiency queue information and the correlation weight of its neighbors, without requiring network-wide information.

\section{Optimality of $(\mathrm{CORN})^{2}$}

In this section, we present the main theorem that shows the optimality of our cooperative sensing scheduling algorithm and establish its performance measures.

We denote by $\left(g_{i}\right)_{i \in \mathcal{N}}$ the cost rate vector (i.e., timeaverage of $\left.\left(G_{i}(t)\right)_{i \in \mathcal{N}}\right)$ induced by a generic sensing schedule $\left(\mu_{i, c}(t)\right)_{i \in \mathcal{N}, c \in \mathcal{C}_{i}}$. Let the feasible cost set, $\mathcal{G}$, be defined as the closure of the set of all cost rate vectors of schedulers satisfying the channel assignment constraint (10). For some small $\epsilon>0$, let $\left(g_{i, \epsilon}^{*}\right) \in \mathcal{G}$ be the minimum cost rate vector with a corresponding scheduler $\left(\mu_{i, c}(t)\right)$ satisfying the following:

$$
\begin{aligned}
\lim _{T \rightarrow \infty} \frac{1}{T} \sum_{t=0}^{T-1} \mu_{i, c}(t) & \geq R_{S}+\epsilon, \\
\text { and } \lim _{T \rightarrow \infty} \frac{1}{T} \sum_{t=0}^{T-1} M_{i, c}(t) & \geq R_{D}+\epsilon .
\end{aligned}
$$

According to [15], $\lim _{\epsilon \rightarrow 0^{+}} \sum_{i \in \mathcal{N}} g_{i, \epsilon}^{*}=\sum_{i \in \mathcal{N}} g_{i}^{*}$ where $\left(g_{i}^{*}\right) \in \mathcal{G}$ is the minimum cost rate vector that satisfies the conditions (6).

\section{A. Optimality of $(C O R N)^{2}$}

The following theorem states the cost optimality and the stability of virtual queues, under the scheduler (9), and under the scheduler (12) if the assumption (11) holds:

Theorem 1: Given $\epsilon>0$, the algorithm can achieve a timeaveraged ensemble cost:

$$
\limsup _{T \rightarrow \infty} \frac{1}{T} \sum_{t=0}^{T-1} \mathbb{E}\left\{G_{i}(t)\right\} \leq \sum_{i \in \mathcal{N}} g_{i, \epsilon}^{*}+\frac{B}{V},
$$

with all the virtual queues bounded by:

$$
\begin{aligned}
& \limsup _{T \rightarrow \infty} \frac{1}{T} \sum_{t=0}^{T-1} \sum_{i \in \mathcal{N}} \sum_{c \in \mathcal{C}_{i}} \mathbb{E}\left\{Q_{i, c}^{D}(t)+Q_{i, c}^{S}(t)\right\} \\
\leq & \frac{V \sum_{i \in \mathcal{N}} g_{i, \epsilon}^{*}+B}{\epsilon}
\end{aligned}
$$

where $B \triangleq \frac{1}{2}\left(R_{D}^{2}+R_{S}^{2}+M_{\max }+1\right) \sum_{i \in \mathcal{N}} \mathcal{C}_{i} .{ }^{2}$

Remark 2: The inequality (14) indicates that the virtual queues are stable, and hence the constraints (6) are satisfied. Since $B$ is independent of the control parameter $V$, the inequality (13) states that the cost of the proposed algorithm can be arbitrarily close to $\sum_{i \in \mathcal{N}} g_{i, \epsilon}^{*}$, when $V$ is chosen large

\footnotetext{
${ }^{2}$ Note that the expectation in $(13)(14)$ is over the initial virtual queue backlogs (i.e., $\left(Q_{i, c}^{S}(0)\right)$ and $\left.\left(Q_{i, c}^{D}(0)\right)\right)$ and possible randomization in (9) and (12) where we break the tie uniformly randomly when necessary.
} 
enough. That is, the algorithm can approach arbitrarily to the optimal cost $\sum_{i \in \mathcal{N}} g_{i}^{*}$ when $\epsilon$ is chosen small enough and $V$ large enough. Note that a smaller value of $\epsilon$ and a larger value of $V$ increase the upper-bound of the virtual queue backlogs (14), and results in slower convergence of the algorithm. We also note that the $O(V)$ upper-bound on the virtual queues is not necessarily tight. Existing works, such as [39][40] (for convex objective functions) [41] (for linear objective functions), have shown stronger result of a universal lower-bound $\Omega(\log V)$ on the queue backlogs. On the other hand, Eryilmaz and Srikant have shown in [42] that $O(V)$ upper-bound is tight in a heavy-traffic scenario.

The proof of Theorem 1 is provided in the next subsection.

\section{B. Proof of Theorem 1}

We present a lemma to assist the proof of Theorem 1 as follows:

Lemma 1: Given $\epsilon>0$ such that $\left(g_{i, \epsilon}^{*}\right) \in \mathcal{G}$, there exists a stationary randomized sensing scheduling algorithm (denoted by STAT) with sensing schedules $\left(\mu_{i, c}^{S T A T}(t)\right)$ independent of virtual queue backlogs, such that the sensing schedules satisfy:

$$
\begin{gathered}
\mathbb{E}\left\{G_{i}^{S T A T}(t)\right\}=g_{i, \epsilon}^{*}, \forall t, \forall i \in \mathcal{N}, \\
\mathbb{E}\left\{\mu_{i, c}^{S T A T}(t)\right\} \geq R_{S}+\epsilon, \text { and } \mathbb{E}\left\{M_{i, c}^{S T A T}(t)\right\} \geq R_{D}+\epsilon,
\end{gathered}
$$

$\forall t, \forall i \in \mathcal{N}, \forall c \in \mathcal{C}_{i}$. Similar formulations of STAT and their proofs have been given in [15], so we omit the proof of Lemma 1 for brevity.

To prove Theorem 1, we introduce a queue vector $\mathbf{Q}(t) \triangleq\left(\left(Q_{i, c}^{D}(t)\right),\left(Q_{i, c}^{S}(t)\right)\right)$ and define the Lyapunov function $L(\mathbf{Q}(t))$ as follows:

$$
L(\mathbf{Q}(t)) \triangleq \frac{1}{2} \sum_{i \in \mathcal{N}} \sum_{c \in \mathcal{C}_{i}}\left[Q_{i, c}^{D}(t)^{2}+Q_{i, c}^{S}(t)^{2}\right] .
$$

We define the corresponding Lyapunov drift by $\Delta(t) \triangleq$ $\mathbb{E}\{L(\mathbf{Q}(t+1))-L(\mathbf{Q}(t))$.

By squaring the virtual queue dynamics (7) and (8), we can derive the following inequality of the Lyapnov drift:

$$
\begin{aligned}
& \Delta(t)+V \sum_{i \in \mathcal{N}} \mathbb{E}\left\{G_{i}(t) \mid \mathbf{Q}(t)\right\} \\
\leq & B+V \sum_{i \in \mathcal{N}} \mathbb{E}\left\{G_{i}(t) \mid \mathbf{Q}(t)\right\} \\
& +\sum_{i \in \mathcal{N}} \sum_{c \in \mathcal{C}_{i}} \mathbb{E}\left\{\left(R_{D}-M_{i, c}(t)\right) \mid \mathbf{Q}(t)\right\} Q_{i, c}^{D}(t) \\
& +\sum_{i \in \mathcal{N}} \sum_{c \in \mathcal{C}_{i}} \mathbb{E}\left\{\left(R_{S}-\mu_{i, c}(t)\right) \mid \mathbf{Q}(t)\right\} Q_{i, c}^{S}(t) .
\end{aligned}
$$

We find an equivalence of (15) by rearranging terms as follows:

$$
\begin{gathered}
\Delta(t)+V \sum_{i \in \mathcal{N}} \mathbb{E}\left\{G_{i}(t) \mid \mathbf{Q}(t)\right\} \\
\leq B+\sum_{i \in \mathcal{N}} \sum_{c \in \mathcal{C}_{i}}\left(R_{S} Q_{i, c}^{S}(t)+R_{D} Q_{i, c}^{D}(t)\right) \\
-\sum_{i \in \mathcal{N}} \sum_{c \in \mathcal{C}_{i}} \mathbb{E}\left\{\mu_{i, c}(t)\left(Q_{i, c}^{S}(t)-V\left(P_{S}+P_{T x}+P_{R x}\left(I_{i}^{c}-1\right)\right)\right)\right. \\
\left.+M_{i, c}(t) Q_{i, c}^{D}(t) \mid \mathbf{Q}(t)\right\}
\end{gathered}
$$

where we employ the following equality by changing order of summation and noting the assumption of fair cooperation:

$$
\sum_{i \in \mathcal{N}} \sum_{c \in \mathcal{C}_{i}} \sum_{j \in \mathcal{I}_{i} \backslash\{i\}} P_{R_{x}} \mu_{j, c}(t)=\sum_{i \in \mathcal{N}} \sum_{c \in \mathcal{C}_{i}} \mu_{i, c}(t) P_{R_{x}}\left(I_{i}^{c}-1\right) .
$$

If the bounded contribution assumption (11) holds, we can further simplify (16) as follows:

$$
\begin{aligned}
& \Delta(t)+V \sum_{i \in \mathcal{N}} \mathbb{E}\left\{G_{i}(t) \mid \mathbf{Q}(t)\right\} \\
& \leq B+\sum_{i \in \mathcal{N}} \sum_{c \in \mathcal{C}_{i}}\left(R_{S} Q_{i, c}^{S}(t)+R_{D} Q_{i, c}^{D}(t)\right) \\
&-\sum_{i \in \mathcal{N}} \sum_{c \in \mathcal{C}_{i}} \mathbb{E}\left\{\mu _ { i , c } ( t ) \left[Q_{i, c}^{S}(t)+\sum_{j \in \mathcal{I}_{i}} w_{i, j}^{c}(t) Q_{j, c}^{D}(t)\right.\right. \\
&\left.\left.\quad-V\left(P_{S}+P_{T x}+P_{R x}\left(I_{i}^{c}-1\right)\right)\right] \mid \mathbf{Q}(t)\right\},
\end{aligned}
$$

with the employment of the following equality:

$$
\begin{aligned}
& \sum_{i \in \mathcal{N}} \sum_{c \in \mathcal{C}_{i}} \sum_{j \in \mathcal{I}_{i}} \mu_{j, c}(t) w_{j, i}^{c}(t) Q_{i, c}^{D}(t) \\
= & \sum_{i \in \mathcal{N}} \sum_{c \in \mathcal{C}_{i}} \mu_{i, c}(t)\left(\sum_{j \in \mathcal{I}_{i}} w_{i, j}^{c}(t) Q_{j, c}^{D}(t)\right),
\end{aligned}
$$

where we have changed the order of summations.

Note that the third term of the Right-Hand Side (RHS) of (16) (and of (17)) is minimized by the algorithm proposed in Section IV-B (Section IV-C) over the set of all feasible algorithms including STAT introduced in Lemma 1. Therefore, we can substitute into the RHS of (15) a stationary randomized algorithm STAT with the cost rate vector $\left(g_{i, \epsilon}^{*}\right)$, and obtain:

$$
\begin{aligned}
& \Delta(t)+V \sum_{i \in \mathcal{N}} \mathbb{E}\left\{G_{i}(t) \mid \mathbf{Q}(t)\right\} \\
\leq & B+V \sum_{i \in \mathcal{N}} g_{i, \epsilon}^{*}-\epsilon \sum_{i \in \mathcal{N}} \sum_{c \in \mathcal{C}_{i}}\left(Q_{i, c}^{S}(t)+Q_{i, c}^{D}(t)\right) .
\end{aligned}
$$

We take the expectation with respect to the distribution of $\mathbf{Q}(t)$ on both sides of (18) and take the time average on $t=$ $0, \cdots, T-1$, which leads to

$$
\begin{aligned}
& \frac{1}{T} \mathbb{E}\{L(\mathbf{Q}(T))\}+\frac{V}{T} \sum_{t=0}^{T-1} \sum_{i \in \mathcal{N}} \mathbb{E}\left\{G_{i}(t)\right\} \\
& \leq B+V \sum_{i \in \mathcal{N}} g_{i, \epsilon}^{*}-\frac{\epsilon}{T} \sum_{t=0}^{T-1} \sum_{i \in \mathcal{N}} \sum_{c \in \mathcal{C}_{i}} \mathbb{E}\left\{Q_{i, c}^{D}(t)+Q_{i, c}^{S}(t)\right\} .
\end{aligned}
$$

By taking limsup of $T$ on both sides of (19), we can prove (13) and (14), respectively.

\section{S- $(\mathrm{CORN})^{2}$ IN DENSEly POPUlated CRNS}

Recall in the (CORN $)^{2}$ described in Section IV, we assume that each node passively receives the broadcast of sensing information from each of its neighbors at a cost of $P_{R_{x}}$. In a densely populated network (e.g., a densely populated sensor network), a node may suffer from a high energy consumption associated with the communication overhead due to excessive cooperation, while already having a sensing quality rate much 
higher than the required rate $R_{D}$. This inefficiency can be addressed by allowing each SU to be selective in receiving neighborhood information. More formally, we introduce a control parameter $v_{j, i}^{c}(t) \in\{0,1\}$ that allows an SU node $i$ to decide whether it receives the sensing information from a neighbor $j$ at time slot $t$. Specifically, we denote by $v_{j, i}^{c}(t)=1, i \neq j$, the event that node $i$ decides to receive the sensing information of node $j$ on channel $c ; v_{j, i}^{c}(t)=0$, otherwise. A node $i$ can reduce its energy consumption by setting $v_{j, i}^{c}(t)=0$ and avoiding unnecessary reception of sensing information from some neighbors $j$. We let $v_{i, i}^{c}(t)=1, \forall t$, since node $i$ 's sensing information is known to itself. Accordingly, the cost for each node $i$ at time slot $t$ is updated as

$G_{i}(t)=\sum_{c \in \mathcal{C}_{i}}\left[\left(P_{S}+P_{T x}\right) \mu_{i, c}(t)+\sum_{j \in \mathcal{I}_{i} \backslash\{i\}} P_{R_{x}} \mu_{j, c}(t) v_{j, i}^{c}(t)\right]$.

We adopt the same problem formulation in Section IV, with the improvement of sensing quality at node $i$ about a channel $c$ updated as

$$
M_{i, c}(t) \triangleq \sum_{j \in \mathcal{I}_{i}} \mu_{j, c}(t) w_{j, i}^{c}(t) v_{j, i}^{c}(t),
$$

where we assume the following bounded contribution assumption to hold:

$$
\sum_{j \in \mathcal{I}_{i}} \mu_{j, c}(t) w_{j, i}^{c}(t) v_{j, i}^{c}(t) \leq M_{i, c}^{\max } .
$$

The corresponding algorithm solution for each time slot $t$ should be optimized over $\left(\mu_{i, c}(t)\right)_{i, c}$ and $\left(v_{j, i}^{c}(t)\right)_{i, j, c}$. In the following, we introduce the distributed $(\mathrm{CORN})^{2}$ adapted to densely populated CRNs.

\section{Selective $(\mathrm{CORN})^{2}\left(\mathrm{~S}-(\mathrm{CORN})^{2}\right)$ :}

Consider a CRN with SUs $i \in \mathcal{N}$. For each time slot $t$, the optimal $v_{j, i}^{c}(t)=v_{j, i}^{c *}(t), \forall j \in \mathcal{I}_{i} \backslash\{i\}, \forall c \in \mathcal{C}_{i}$, is determined as

$$
v_{j, i}^{c *}(t)=\left\{\begin{array}{l}
1, \text { if } w_{j, i}^{c}(t) Q_{i, c}^{D}(t)>V P_{R_{x}}, \\
0, \text { otherwise. }
\end{array}\right.
$$

The sensing schedules $\left(\mu_{i, c}(t)\right)_{i, c}$ are then determined subject to (10) by

$$
\begin{aligned}
\max _{\left(\mu_{i, c}(t)\right)_{i, c}} & \sum_{i \in \mathcal{N}} \sum_{c \in \mathcal{C}_{i}} \mu_{i, c}(t)\left\{Q_{i, c}^{S}(t)\right. \\
& -V\left(P_{S}+P_{T_{x}}\right)+w_{i, i}^{c}(t) Q_{i, c}^{D}(t) \\
& \left.+\sum_{j \in \mathcal{I}_{i} \backslash\{i\}}\left[w_{i, j}^{c}(t) Q_{j, c}^{D}(t)-V P_{R_{x}}\right]^{+}\right\} .
\end{aligned}
$$

The S-(CORN $)^{2}$ decouples the scheduling of $\left(v_{j, i}^{c}(t)\right)_{i, j, c}$ and $\left(\mu_{i, c}(t)\right)_{i, c}:$ (20) can be solved independently of $\left(\mu_{i, c}(t)\right)_{i, c}$. Specifically, according to (20), SU $i$ decides to receive sensing information from its neighbor $j$ (i.e., $v_{j, i}^{c}(t)=1$ ) when the sensing deficiency of SU $i$ outweighs the energy consumption of receiving SU $j$ 's sensing outcome by a factor of $V$. Similar to the original $(\mathrm{CORN})^{2},(21)$ is a maximal weight matching optimization that can be solved in a greedy and distributed manner. We can show that Theorem 1 holds with $\left(g_{i, \epsilon}^{*}\right)_{i}$ denoting the cost vector $\epsilon$-close to the optimality over all feasible schedules $\left(\left(\mu_{i, c}(t)\right)_{i, c},\left(v_{j, i}^{c}(t)\right)_{i, j, c}\right)$. The corresponding proof for the optimality of $\mathrm{S}-(\mathrm{CORN})^{2}$ can be developed similar as in Section V-B, and we provide a brief proof sketch in the following.

Proof sketch of Optimality: Similar to deriving (17) in the proof of Theorem 1, we observe the following inequality on the Lyapunov drift:

$$
\begin{aligned}
& \Delta(t)+V \sum_{i \in \mathcal{N}} \mathbb{E}\left\{G_{i}(t) \mid \mathbf{Q}(t)\right\} \\
\leq & B+\sum_{i \in \mathcal{N}} \sum_{c \in \mathcal{C}_{i}}\left(R_{S} Q_{i, c}^{S}(t)+R_{D} Q_{i, c}^{D}(t)\right) \\
- & \sum_{i \in \mathcal{N}} \sum_{c \in \mathcal{C}_{i}} \mathbb{E}\left\{\mu _ { i , c } ( t ) \left[Q_{i, c}^{S}(t)+\sum_{j \in \mathcal{I}_{i}} w_{i, j}^{c}(t) v_{i, j}^{c}(t) Q_{j, c}^{D}(t)\right.\right. \\
& \left.\left.-V\left(P_{S}+P_{T x}+P_{R x} \sum_{j \in \mathcal{I}_{i} \backslash\{i\}} v_{i, j}^{c}(t)\right)\right] \mid \mathbf{Q}(t)\right\},
\end{aligned}
$$

where we employ the following two equalities

$$
\begin{aligned}
\sum_{i \in \mathcal{N}} \sum_{c \in \mathcal{C}_{i}} \sum_{j \in \mathcal{I}_{i} \backslash\{i\}} \mu_{j, c}(t) v_{j, i}^{c}(t)=\sum_{i \in \mathcal{N}} \sum_{c \in \mathcal{C}_{i}} \sum_{j \in \mathcal{I}_{i} \backslash\{i\}} \mu_{i, c}(t) v_{i, j}^{c}(t), \\
\sum_{i \in \mathcal{N}} \sum_{c \in \mathcal{C}_{i}} \sum_{j \in \mathcal{I}_{i}} \mu_{j, c}(t) w_{j, i}^{c}(t) v_{j, i}^{c} Q_{i, c}^{D}(t) \\
=\sum_{i \in \mathcal{N}} \sum_{c \in \mathcal{C}_{i}} \mu_{i, c}(t)\left(\sum_{j \in \mathcal{I}_{i}} w_{i, j}^{c}(t) v_{i, j}^{c}(t) Q_{j, c}^{D}(t)\right),
\end{aligned}
$$

by changing the order of summation. The last term on the RHS of (22) is equivalent to

$$
\begin{aligned}
-\sum_{i \in \mathcal{N}} & \sum_{c \in \mathcal{C}_{i}} \mathbb{E}\left\{\mu _ { i , c } ( t ) \left[Q_{i, c}^{S}(t)\right.\right. \\
& -V\left(P_{S}+P_{T x}\right)+w_{i, i}^{c}(t) Q_{i, c}^{D}(t) \\
& \left.\left.+\sum_{j \in \mathcal{I}_{i} \backslash\{i\}} v_{i, j}^{c}(t)\left(w_{i, j}^{c}(t) Q_{j, c}^{D}(t)-V P_{R x}\right)\right] \mid \mathbf{Q}(t)\right\}
\end{aligned}
$$

where we make use of the fact that $v_{i, i}^{c}(t)=1, \forall i, \forall c$.

It is easy to check that (20) (21) in S-(CORN) $)^{2}$ minimizes (23), and hence, minimizes the RHS of (22), over a group of algorithms including STAT introduced in Lemma 1 (with the corresponding sensing schedule $\left(\left(\mu_{i, c}^{S T A T}(t)\right)_{i, c},\left(v_{j, i}^{c, S T A T}(t)\right)_{i, j, c}\right)$. By substituting STAT to the RHS of (22) and following the proof in deriving (19), we can prove the optimality of S-(CORN) ${ }^{2}$.

We note that in the literature of network resource scheduling, a Lyapunov optimization approach leads to a centralized high-complexity solution in [15][16] and a random access approach in [36][37][38] achieves a distributed optimal solution with the overheads of RTS/CTS handshake. In this work, we have shown under the proposed virtual queue framework and under mild assumption (11) (which is easily satisfied when we set $M_{i, c}^{\max }$ large enough) that our proposed near optimal solutions of $(\mathrm{CORN})^{2}$ and $\mathrm{S}-(\mathrm{CORN})^{2}$ are distributed with low complexity without the overheads of RTS/CTS.

\section{NumERicAl EVALUATIONS}

In this section, using numerical analyses via Matlab, we evaluate $(\mathrm{CORN})^{2}$ (discussed in Section IV-B) in Section VII-A and compare the performance of the distributed version of $(\mathrm{CORN})^{2}$ and the distributed S-(CORN $)^{2}$ in Section VII-B. 
For the evaluations, a CRN of $N$ SU nodes operating on a single channel is considered, where each node tries to estimate the spectrum occupancy on the channel using either noncooperative or cooperative sensing employing $(\mathrm{CORN})^{2}$.

Non-cooperative sensing is modeled as a special case of $(\mathrm{CORN})^{2}$. The sensing deficiency queue, $Q_{i, c}^{D}(t)$, is not considered and the spectrum sensing is scheduled according to only the local sensing queue, $Q_{i, c}^{S}(t)$. In a non-cooperation case, a node $i$ improves its sensing quality about channel $c$ for any time slot $t$ by:

$$
M_{i, c}(t) \triangleq \min \left\{\sum_{j \in \mathcal{I}_{i}} \mu_{j, c}(t) w_{j, i}^{c}(t), M_{i, c}^{\max }\right\}=\mu_{j, c}(t),
$$

where we used the fact that $w_{i, i}^{c}(t)=1$ and $M_{i, c}^{\max }>1$ from Section IV. Hence, with non-cooperative sensing, the local sensing rate is set to the maximum of the $R_{D}$ and $R_{S}$ parameters used in the cooperative sensing case for fairness. Moreover, the energy consumption cost is $G_{i}(t)=\sum_{c \in \mathcal{C}_{i}} P_{s} \mu_{i, c}(t)$ at each node $i$, i.e., cooperation overhead is not considered. In our numerical evaluations, the cost represents energy consumption associated with sensing. In all tests, we use the following parameters: $P_{S}=3.5 \mathrm{~mJ}, P_{T x}=P_{R x}=0.1125 \mathrm{~mJ}$, which are consistent with the values reported in [24]. For cooperative sensing, all $N$ nodes are assumed to be available for cooperation, i.e., $I_{i}=N, \forall i \in \mathcal{N}$. The maximum level of information that can be obtained in a single time slot is limited to 2, i.e., $M_{i, c}^{\max }=2, \forall i \in \mathcal{N}$, and the channel $c$ is considered. For notation simplicity, $w_{i, j}^{c}(t)$ is replaced by $w_{i, j}(t)$ in the following analysis. Further to simplify the algorithm evaluation, we assume in this section that $w_{i, j}(t)$ is fixed over time $t$ and uniform over $i, j \in \mathcal{N}$ with $i \neq j$ (i.e., $w_{i, j}(t)=w_{i^{\prime}, j^{\prime}}(t), \forall i, j, i^{\prime}, j^{\prime} \in \mathcal{N}$ with $i \neq j$ and $\left.i^{\prime} \neq j^{\prime}\right)$. Each data point represents the average values observed over 10,000 simulated time slots. In the simulations, we set the control parameter $V=10$, since we observe that a larger value of $V$ cannot noticeably decrease the energy consumption but results in a slower convergence of the algorithm.

\section{A. $(C O R N)^{2}$ Performance}

In Fig. 2(a), the energy consumption per node per time slot is shown as a function of the number of nodes in the network under $(\mathrm{CORN})^{2}$ and the non-cooperative sensing case. The energy consumption performance is investigated for different rate of sensing quality, $R_{D}$, values and $R_{S}=0.55$. It can be observed that cooperative sensing with $(\mathrm{CORN})^{2}$ improves energy consumption compared to non-cooperative sensing when the number of nodes exceeds three, mainly owing to the fact that cost of cooperation is offset by its benefits for larger networks. For small clusters, $R_{D}$ is observed to have a negative impact on the energy efficiency, where the energy consumption of cooperative sensing can be as much as $75 \%$ higher than that of non-cooperative sensing. On the other hand, as the number of cooperating nodes increases, $R_{S}$ dominates scheduling decisions since satisfying local sensing queue constraints becomes sufficient to satisfy any sensing deficiency queue constraints.

We next investigate the effect of the correlation weights among neighboring nodes on the energy optimality. The local correlation values are assumed to be unity, i.e., $w_{i, i}(t)=1$. In Fig. 2(b), the energy consumption per node per time slot is shown as a function of the number of nodes for different correlation levels. The non-cooperative case is also shown. The results indicate that $(\mathrm{CORN})^{2}$ is able to save energy compared to the non-cooperative case and the savings increase with higher correlation levels. Perfect correlation results in the highest cost saving. When the correlation levels are sufficiently low, the cost of cooperation dominates benefits reaped. In the pathological case of no correlation, the cost increases with the number of nodes due to the increased cost of sensing information reception. However, this information does not benefit the receivers in terms of sensing information accuracy and results in a larger energy consumption than the non-cooperative case. These results emphasize the fact that cooperation is not beneficial in every case, especially when the correlation between nodes is very limited and the energy consumption for communication is higher than that for sensing.

\section{B. Distributed $(C O R N)^{2}$ and $S-(C O R N)^{2}$}

In this section, we compare the three distributed sensing algorithms: the distributed cooperative solution of $(\mathrm{CORN})^{2}$ (discussed in Section IV-C), the cooperative S-(CORN) ${ }^{2}$ (discussed in Section VI), and the non-cooperative sensing case. Specifically, their energy consumption performance against $P_{T x}=P_{R x}$ (the energy consumption for broadcasting $\left(P_{T x}\right)$ and receiving $\left(P_{R x}\right)$ sensing information) is shown in Fig. 3(a), where the sensing cost is fixed as $P_{S}=3.5 \mathrm{~mJ}$, the number of nodes is set as $N=9$, and $M_{i, c}^{\max }=10, \forall i$, $\forall c$. It can be observed that $\mathrm{S}-(\mathrm{CORN})^{2}$ always outperforms $(\mathrm{CORN})^{2}$, since SUs can decide whether or not to receive sensing information of neighboring nodes (and hence save energy consumption) under S-(CORN) ${ }^{2}$. We can also observe in Fig. 3(a) that the saved energy consumption becomes much larger under $\mathrm{S}-(\mathrm{CORN})^{2}$ when the cost of communication increases. In cases where communication is cheaper than sensing (i.e., $P_{T x}$ is comparatively smaller than $P_{S}$ ), cooperative sensing outperforms non-cooperative sensing with diminishing returns. For higher values of $P_{T x}$ (i.e., from energy consumption perspective, when communication is expensive), the energy consumption of non-cooperative sensing can be smaller than cooperative sensing. Hence, through Fig. 3(a), we have shown that the cost for communication with respect to sensing is an important factor trading off between local sensing and cooperation. Compared to $(\mathrm{CORN})^{2}, \mathrm{~S}-(\mathrm{CORN})^{2}$ can accommodate larger communication energy levels, while still outperforming non-cooperative communication.

We also investigate the effect of the number of users on energy consumption in Fig. 3(b). As the number of users initially increases, a full cooperation is encouraged as the energy consumption decreases significantly. That is why the distributed solution of $(\mathrm{CORN})^{2}$ and $\mathrm{S}-(\mathrm{CORN})^{2}$ coincide in energy consumption at $N=2,3$. As the number of users increases, the communication cost increases, leading to an increase in energy consumption. We also note that when the number of users increases, some users may decide not to receive the broadcasted sensing information from neighbors 


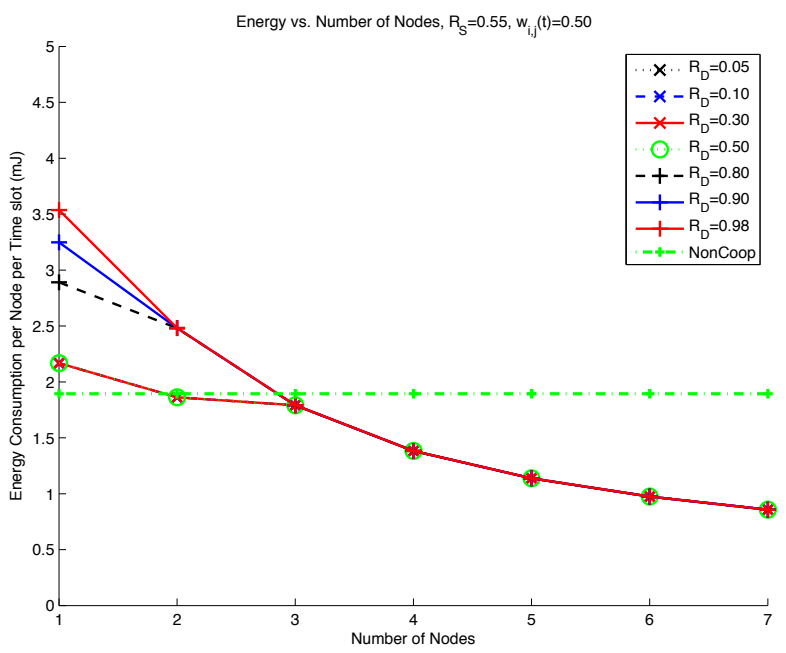

(a)

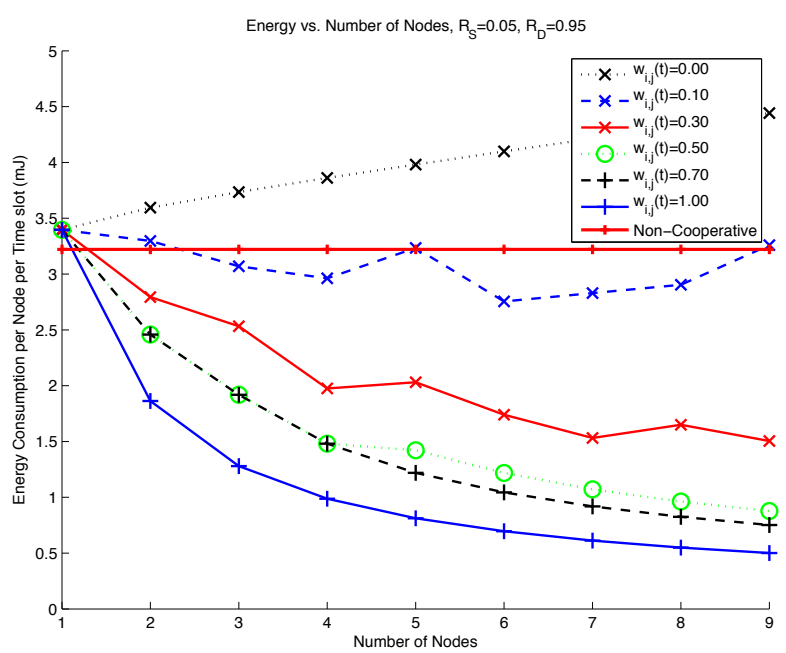

(b)

Fig. 2. Optimal energy consumption vs. number of nodes for (a) different sensing quality rates and (b) different correlation weights among neighbors.

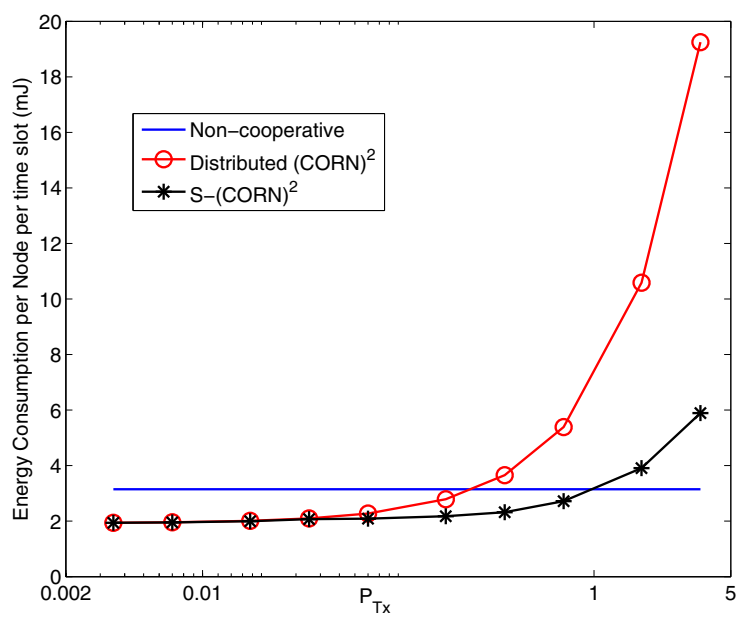

(a) Energy consumption vs. $P_{T x}=P_{R x}, N=9, R_{S}=0.55$

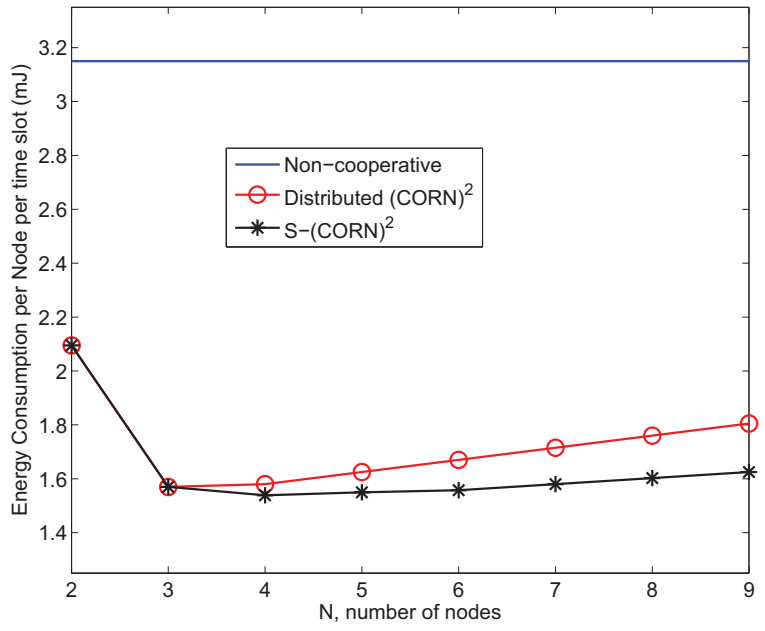

(b) Energy consumption vs. $N, P_{T x}=P_{R x}=0.1125 \mathrm{~mJ}, R_{S}=0.4$

Fig. 3. Energy consumption vs. (a) $P_{T x}$ and (b) number of nodes $N . R_{D}=0.9, w_{i j}(t)=0.6$.

under S-(CORN) ${ }^{2}$ (i.e., excessive cooperation is avoided). This results in a lower energy consumption than the original $(\mathrm{CORN})^{2}$. Thus, as the number of users increases (i.e., as the SU network becomes populated), the increase in energy consumption under $\mathrm{S}-(\mathrm{CORN})^{2}$ is not as sharp as that under $(\mathrm{CORN})^{2}$.

\section{Comparisons with Spatial-Correlation Based Sensing Scheduling Algorithms}

In this section, we compare $(\mathrm{CORN})^{2}$ with respect to a spatial-correlation-based cooperative sensing algorithm, where we do not require a minimum sensing rate constraint (i.e., $\left.R_{S}=0\right)$. Different from $(\mathrm{CORN})^{2}$, the spatial-correlationbased algorithm is developed to minimize the energy consumption employing spatial correlations only. ${ }^{3}$ Specifically,

${ }^{3}$ This spatial-correlation-based algorithm is inspired by and based in part on [10]. Note that we have considered local sensing quality constraint in this algorithm instead of the global constraint on probability of misdetection/false alarm in [10]. the centralized spatial-correlation-based algorithm is defined as follows, for each time slot $t$,

$$
\begin{aligned}
& \operatorname{minimize} \sum_{i \in \mathcal{N}} G_{i}(t) \\
& \text { subject to } \sum_{j \in \mathcal{I}_{i}} \mu_{j, c}(t) w_{j, i}^{c}(t) \geq R_{D}, \forall i \in \mathcal{N}, c \in \mathcal{C}_{i},
\end{aligned}
$$

with constraint (10). Under the above spatial-correlation-based algorithm, at each time slot $t$, if an $\mathrm{SU}$ is scheduled to sense a channel, it will broadcast its sensing data to its neighbors. However, an SU $i$ does not utilize its neighbor SU $j$ 's sensing data if $\mathrm{SU} j$ is not scheduled to perform sensing at the current time slot $t$ (i.e., if $\mu_{j, c}(t)=0$ ), as is captured by the constraint (24). That is, under this algorithm a node does not utilize any sensing information before the current time slot (either from its local sensing history or its neighbors broadcasted data in the past). Hence, this algorithm optimizes the total energy consumption only based on the current spatial 


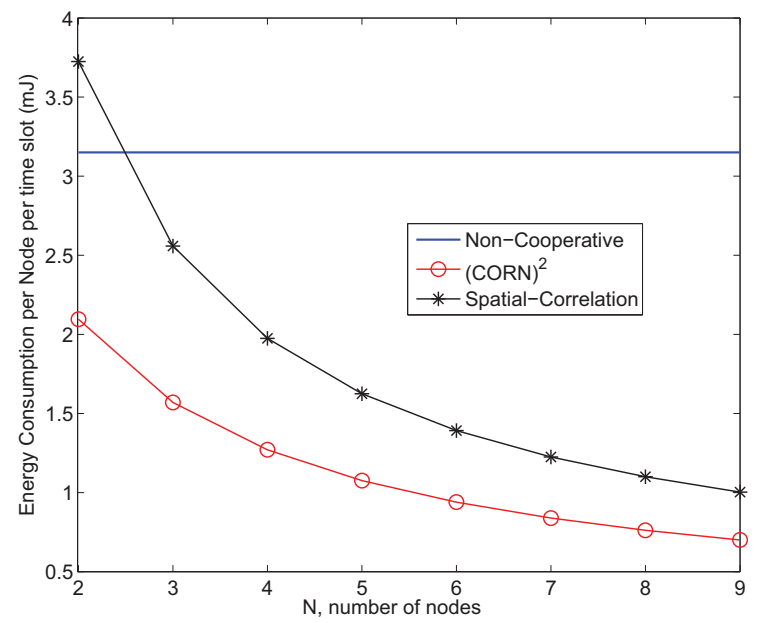

Fig. 4. Energy consumption vs. number of nodes $N . R_{D}=0.9, R_{S}=0$, $w_{i j}(t)=0.6$.

correlations. ${ }^{4}$ We also note that this algorithm is required to solve a centralized optimization, while $(\mathrm{CORN})^{2}$ can be solved in a distributed fashion under the bounded contribution assumption.

With the same simulation setup as introduced at the beginning of Section VII and $R_{D}=0.9$, the (per node per time slot) energy consumption is shown in Figure 4 under $(\mathrm{CORN})^{2}$, the spatial-correlation-based algorithm, and the non-cooperative algorithm. It can be observed that $(\mathrm{CORN})^{2}$ indeed outperforms the spatial-correlation-based algorithm and the non-cooperative algorithm under the same number of SU nodes. We also observe that when the number of nodes is small (e.g., $N=2$ ), the gain from spatial correlation alone cannot compensate for the additional cooperative communication cost, compared to the non-cooperative sensing algorithm.

\section{CONCLUSIONS}

In this paper, we studied the problem of cooperative spectrum sensing, where we have leveraged the spatio-temporal correlations between spectral observations among different nodes and across different time points. Based on the notion of sensing information accuracy, which decays with time, we have developed virtual queue structures that represent the evolution of sensing quality in a given node. These virtual queues form the basis of our novel sensing scheduling algorithms that minimize the total cost of spectrum sensing while guaranteeing given levels of average sensing quality. The developed algorithm and its variants are theoretically shown to minimize the sensing cost and stabilizing all queues in the network, which in turn guarantees desired sensing quality levels. In our future work, we will extend the sensing algorithm both theoretically and in implementations to the case

\footnotetext{
${ }^{4}$ In comparison, the past sensing information is captured by the total sensing deficiency queue $Q_{i, c}^{D}(t)$ under $(\text { CORN })^{2}$ : Node $i$ receives the spectrum estimate from nodes $j$ (which sense the same channel at time $t$ ) and this contributes to the spectrum sensing knowledge and decreases the virtual queue by $M_{i, c}(t)$ with respect to spatial correlation. As time goes by, the virtual queue increases by $R_{D}$, which captures the loss in spectrum information quality as the temporal correlation decreases.
}

where $R_{D}$ changes over SUs and channels under different mobility models.

\section{REFERENCES}

[1] S. H. Ahmad, M. Liu, T. Javadi, Q. Zhao, and B. Krishnamachari, "Optimality of myopic sensing in multi-channel opportunistic access," IEEE Trans. Inf. Theory, 2009.

[2] I. F. Akyildiz, W. Y. Lee, M. C. Vuran, and S. Mohanty, "NeXt generation/dynamic spectrum access/cognitive radio wireless networks: a survey," Comput. Netw., vol. 50, no. 13, pp. 2127-2159, 2006.

[3] I. F. Akyildiz, W.-Y. Lee, M. C. Vuran, and S. Mohanty, "A survey on spectrum management in cognitive radio networks," IEEE Commun. Mag., vol. 46, no. 4, pp. 40-48, Apr. 2008.

[4] A. Fehske, J. Gaeddert, and J. H. Reed, "A new approach to signal classification using spectral correlation and neural networks," in Proc. 2005 IEEE DYSPAN, pp. 144-150.

[5] H. Kopka and P. W. Daly, "Cluster-based spectrum sensing architecture for opportunistic spectrum access networks," Delft University of Technology, Tech. Rep. IRCTR-S-004-07, 2006.

[6] W.-Y. Lee and I. F. Akyildiz, "Optimal spectrum sensing framework for cognitive radio networks," IEEE Trans. Wireless Commun., vol. 7, no. 10, pp. 3845-3857, Oct. 2008.

[7] J. Lunden, V. Koivunen, A. Huttunen, and V. H. Poor, "Spectrum sensing in cognitive radios based on multiple cyclic frequencies," in Proc. 2007 Crown Com, pp. 37-43.

[8] H. Hu, Y. Wang, J. Song, "Signal classification based on spectral correlation analysis and SVM in cognitive radio," in Proc. 2008 AINA, pp. 883-887.

[9] B. L. Mark and A. O. Nasif, "Estimation of maximum interference-free transmit power lever for opportunistic spectrum access," IEEE Trans. Wireless Commun., 2009.

[10] A. W. Min and K. G. Shin, "An optimal sensing framework based on spatial RSS-profile in cognitive radio networks," in Proc 2009 IEEE SECON.

[11] H. N. Pham, Y. Zhang, P. E. Engelstad, T. Skeie, and F. Eliassen, "Optimal cooperative spectrum sensing in cognitive sensor networks," in Proc. 2009 IWCMC.

[12] E. Visotsky, "On collaborative detection of TV transmissions in support of dynamic spectrum sensing," in Proc. 2005 IEEE Symp. Dynamic Spectrum Access Netw., pp. 338-345.

[13] W. Xia, S. Wang, W. Liu, and W. Cheng, "Correlation-based spectrum sensing in cognitive radio," in Proc. 2009 ACM Workshop Cognitive Radio Netw.

[14] L. Tassiulas and A. Ephremides, "Stability properties of constrained queueing systems and scheduling policies for maximum throughput in multihop radio networks," IEEE Trans. Autom. Control, vol. 37, no. 12, pp. 1936-1948, Dec. 1992.

[15] M. Neely, "Energy optimal control for time varying wireless networks," IEEE Trans. Inf. Theory, vol. 52, no. 7, pp. 2915-2934, July 2006.

[16] L. Georgiadis, M. Neely, and L.Tassiulas, "Resource allocation and cross-layer control in wireless networks," Foundations Trends Netw., pp. 1-149, 2006.

[17] A. Eryilmaz, R. Srikant, and J. Perkins, "Stable scheduling policies for fading wireless channels," IEEE/ACM Trans. Netw., vol. 13, no. 2, pp. 411-424, Apr. 2005.

[18] A. Eryilmaz and R. Srikant, "Joint congestion control, routing and MAC for stability and fairness in wireless networks," IEEE J. Sel. Areas Commun., vol. 24, no. 8, pp. 1514-1524, Aug. 2006.

[19] X. Wu, R. Srikant, and J. Perkins, "Scheduling efficiency of distributed greedy scheduling algorithms in wireless networks," IEEE Trans. Mobile Comput., vol. 6, no. 6, pp. 595-605, June 2007.

[20] P. Chaporkar, K. Kar, and S. Sarkar, "Throughput guarantees through maximal scheduling in wireless networks," in Proc. 2005 Annual Allerton Conf. Commun., Control, Comput., pp. 1-11.

[21] C. Joo, X. Lin, and N. Shroff, "Greedy maximal matching: performance limits for arbitrary network graphs under the node-exclusive interference model," IEEE Trans. Automatic Control, vol. 54, no. 12, pp. 2734-2744, Dec. 2009.

[22] X. Lin and S. Rasool, "A distributed joint channel-assignment, scheduling and routing algorithm for multi-channel ad hoc wireless networks," in Proc. 2007 IEEE INFOCOM, pp. 1118-1126.

[23] D. Xue and E. Ekici, "Optimal power allocation in multi-hop wireless networks with finite buffers," in Proc. 2010 IEEE ICC, pp. 1-5.

[24] V. Namboodiri, "Are cognitive radios energy efficient? A study of the wireless LAN scenario," in Proc. 2009 IEEE IPCCC, pp. 437-442. 
[25] T. Do and B. L. Mark, "Joint spatial-temporal spectrum sensing for cognitive radio networks," IEEE Trans. Veh. Technol., vol. 59, no. 7 pp. 3480-3490, Sept. 2010.

[26] R. Tandra, A. Sahai, and V. V. Veeravalli, "Space-time metrics for spectrum sensing," in Proc. 2010 IEEE Symp. New Frontiers Dynamic Spectrum, pp. $1-12$

[27] D. Cabric, A. Tkachenko, and R. W. Brodersen, "Experimental study of spectrum sensing based on energy detection and network cooperation," in Proc. 2006 ACM Int. Workshop Technol. Policy Accessing Spectrum.

[28] A. Ghasemi and E. S. Sousa, "Asymptotic performance of collaborative spectrum sensing under correlated log-normal shadowing," IEEE Commun. Lett., vol. 11, no. 1, pp. 34-36, Jan. 2007.

[29] M. C. Vuran and O. B. Akan, "Spatio-temporal characteristics of point and field sources in wireless sensor networks," in Proc. 2006 IEEE Int Conf. Commun., pp. 234-239.

[30] Y. Selen, H. Tullberg, and J. Kronander, "Sensor selection for cooperative spectrum sensing," in Proc. 2008 IEEE Symp. New Frontiers Dynamic Spectrum Access Netw., pp. 1-11.

[31] M. Di Renzo, F. Graziosi, and F. Santucci, "Cooperative spectrum sensing in cognitive radio networks over correlated log-normal shadowing," in Proc. 2009 IEEE Veh. Technol. Conf., pp. 1-5.

[32] M. Gudmundson, "Correlation model for shadow fading in mobile radio systems," Electron. Lett., vol. 27, no. 23, pp. 2145-2146, Nov. 1991.

[33] A. W. Min, K. G. Shin, and X. Hu, "Secure cooperative sensing in IEEE 802.22 WRANs using shadow fading correlation," IEEE Trans. Mobile Comput., vol. 10, no. 10, pp. 1434-1447, Oct. 2011.

[34] I. Forkel, M. Shinnenburg, and M. Ang, "Generation of two-dimensional correlated shadowing for mobile radio network simulation," in Proc. 2004 Int. Symp. Wireless Personal Multimedia Commun.

[35] J. Beek, J. Riihijarvi, A. Achtzehn, and P. Mahonen, "UHF white space in Europe - a quantitative study into the potential of the $470-790 \mathrm{MHz}$ band," in Proc. 2011 IEEE DySPAN, pp. 1-9.

[36] L. Jiang and J. Walrand, "A distributed algorithm for throughput and utility maximization in wireless networks," IEEE/ACM Trans. Netw., vol. 18 , no.3, pp. 960-972, June 2010.

[37] S. Rajagopalan, D. Shah, and J. Shin, "Network adiabatic theorem: an efficient randomized protocol for contention resolution," in Proc. 2009 International Joint Conf. Measurement Modeling Comput. Syst., pp. 133-144.

[38] J. Ni, B. Tan, and R. Srikant, "Q-CSMA: queue-length based CSMA/CA algorithms for achieving maximum throughput and low delay in wireless networks," in Proc. 2010 IEEE INFOCOM Mini-Conf.

[39] R. Berry and R. Gallager, "Communication over fading channels with delay constraints," IEEE Trans. Inf. Theory, vol. 48, no. 5, pp. 11351149, May 2002.

[40] M. Neely, "Intelligent packet dropping for optimal energy-delay tradeoffs in wireless downlinks," IEEE Trans. Automatic Control, vol. 54, no. 3, pp. 565-579, Mar. 2009.

[41] B. Tan and R. Srikant, "Online advertisement, optimization and stochastic networks," IEEE Trans. Automatic Control, vol. 57, no. 11, Nov. 2012.

[42] A. Eryilmaz and R. Srikant, "Asymptotically tight steady-state queue length bounds implied by drift conditions," Queueing Syst.: Theory Applications, vol. 72, no. 3-4, pp. 311-359, Dec. 2012.

[43] A. Min and K. Shin, "Impact of mobility on spectrum sensing in cognitive radio networks," in Proc. 2009 ACM Workshop Cognitive Radio Netw.), pp. 13-18.

[44] F. Yu, H. Tang, M. Huang, P. Mason, and Z. Li, "Distributed cooperative spectrum sensing in mobile ad hoc networks with cognitive radios," Comput. Commun., vol. 36, no. 12, pp. 1341-1349, July 2013.

[45] Z. Li, R. Yu, and M. Huang, "A distributed consensus-based cooperative spectrum-sensing scheme in cognitive radios," IEEE Trans. Veh. Technol., vol. 59, no. 1, pp. 383-393, Jan. 2010.
[46] S. Huang, X. Liu, and Z. Ding, "Optimal transmission strategies for dynamic spectrum access in cognitive radio networks," in IEEE Trans. Mobile Comput., vol. 8, no. 12, pp. 1636-1648, Dec. 2009.

[47] M. Felice, K. Chowdhury, and L. Bononi, "Cooperative spectrum management in cognitive vehicular ad hoc networks," in Proc. 2011 IEEE Veh. Netw. Conf., pp. 47-54.

[48] S. Tang and J. Yuan, "DAMson: on distributed sensing scheduling to achieve high quality of monitoring," in Proc. 2013 IEEE Infocom MiniConf., pp. 155-159.

[49] A. Min, K. Kim, J. Singh, and K. Shin, "Opportunistic spectrum access for mobile cognitive radios," in Proc. 2011 Infocom, pp. 2993-3001.

[50] Y. Huang and X. Huang, "Detection of temporally correlated signals over multipath fading channels," IEEE Trans. Wireless Commun., vol. 12, no. 3, pp. 1536-1276, Mar. 2013.

[51] D. Xue, E. Ekici, and M. C. Vuran, " $(\mathrm{CORN})^{2}$ : correlation-based cooperative spectrum sensing in cognitive radio networks," in Proc. 2012 WiOpt, pp. 161-168.

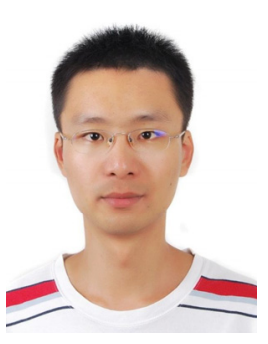

Dongyue Xue (S'11) received his B.S. degree in Information Engineering from Shanghai Jiaotong University, Shanghai, China, in 2009, and received his M.S. and Ph.D. degrees in Electrical and Computer Engineering at The Ohio State University, Columbus, OH, in 2013. His research interests include cross-layer scheduling in wireless networks and dynamic resource allocation in cognitive radio networks.

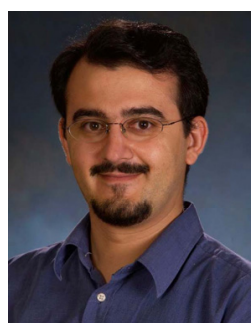

Eylem Ekici (S'99-M'02-SM'11) received his BS and MS degrees in Computer Engineering from Bogazici University, Istanbul, Turkey, in 1997 and 1998, respectively, and the $\mathrm{PhD}$ degree in Electrical and Computer Engineering from the Georgia Institute of Technology, Atlanta, GA, in 2002. Currently, he is an Associate Professor with the Department of Electrical and Computer Engineering, The Ohio State University. His current research interests include wireless networks, vehicular communication systems, cognitive radio networks, nano communication systems, with an emphasis on resource management, and analysis of network architectures and protocols. He is an Associate Editor of IEEE/ACM TRANSACTIONS ON NETWORKING, IEEE TRANSACTIONS on Mobile Computing, Computer Networks Journal (Elsevier) and ACM Mobile Computing and Communications Review.

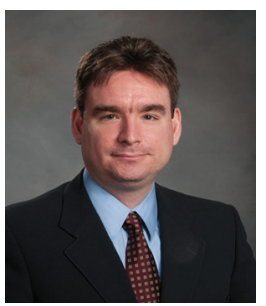

Mehmet C. Vuran (S'98-M'07) received his B.Sc. degree in Electrical and Electronics Engineering from Bilkent University, Ankara, Turkey, in 2002. He received his M.S. and Ph.D. degrees in Electrical and Computer Engineering from the Georgia Institute of Technology, Atlanta, in 2004 and 2007, respectively, under the guidance of Prof. Ian F. Akyildiz. Currently, he is an Associate Professor in the Department of Computer Science and Engineering at the University of Nebraska-Lincoln and Robert B. Daugherty Water for Food Institue Fellow. He is the recipient of an NSF CAREER award in 2010 and the co-author of Wireless Sensor Networks textbook. His current research interests include cognitive radio networks, cross-layer design and correlation-based communication for wireless sensor networks, and wireless underground communications. $\mathrm{He}$ is an Associate Editor of IEEE TRANS ACTIONS ON WIRELESS COMMUNICATIONS, Computer Networks Journal (Elsevier), IEEE INTERNET OF THINGS JOURNAL, and IEEE COMMUNICATIONS SURVEYS AND TUTORIALS. 